Ephemeride des Kometen $1904 \mathrm{I}$.

I $2^{\text {h }}$ M. Z. Greenwich. Fortsetzung von A. N. 3986 .

\begin{tabular}{|c|c|c|c|c|c|c|c|}
\hline \multicolumn{2}{|c|}{1905} & \multicolumn{2}{|r|}{$\alpha$} & \multicolumn{2}{|c|}{$\delta$} & $\log A$ & $\mathrm{H}$ \\
\hline \multirow{15}{*}{ Mai } & 2 & $7^{h} 5$ & $7^{\mathrm{mn}} 16^{\mathrm{s}}$ & $+53^{\circ}$ & $9: 0$ & 0.7114 & 0.059 \\
\hline & 4 & & $\{7$ I 3 & $5^{2}$ & $49 \cdot 3$ & & \\
\hline & 6 & & $7 \quad I 4$ & $5^{2}$ & 29.9 & 0.7187 & 0.056 \\
\hline & 8 & & 720 & $5^{2}$ & 10.9 & & \\
\hline & IO & & 730 & 51 & $5^{2.2}$ & $0.725^{8}$ & 0.053 \\
\hline & I 2 & & 744 & $5 \mathrm{I}$ & 33.8 & & \\
\hline & $x_{4}$ & & 82 & 51 & I 5.7 & 0.7326 & 0.050 \\
\hline & 16 & & $8 \quad 24$ & 50 & 58.0 & & \\
\hline & I 8 & & $8 \quad 49$ & 50 & 40.6 & 0.7392 & 0.048 \\
\hline & 20 & & 917 & 50 & 23.5 & & \\
\hline & 22 & 7 & $94^{8}$ & $5^{\circ}$ & 6.7 & 0.7455 & 0.046 \\
\hline & 24 & & 022 & 49 & 50.2 & & \\
\hline & 26 & & - $5^{8}$ & 49 & $34 . x$ & 0.7516 & 0.044 \\
\hline & 28 & & I 37 & 49 & I 8.3 & & \\
\hline & 30 & & $2 \quad 18$ & 49 & 2.8 & 0.7574 & 0.042 \\
\hline \multirow[t]{15}{*}{ Juni } & I & & $3 \mathrm{I}$ & 48 & 47.6 & & \\
\hline & 3 & & 346 & 48 & 32.8 & 0.7630 & $0.04 \mathrm{I}$ \\
\hline & 5 & & 433 & 48 & I 8.2 & & \\
\hline & 7 & & 522 & 48 & 3.9 & 0.7683 & 0.040 \\
\hline & 9 & & 612 & 47 & 49.9 & & \\
\hline & I I & & 74 & 47 & $3^{6.2}$ & 0.7733 & 0.039 \\
\hline & $x_{3}$ & & 757 & 47 & 22.7 & & \\
\hline & I 5 & & 852 & 47 & 9.5 & 0.7780 & 0.038 \\
\hline & I 7 & & 948 & 46 & $5^{6.6}$ & & \\
\hline & 19 & & 045 & 46 & 44.0 & 0.7824 & 0.037 \\
\hline & $2 \mathrm{I}$ & & I 43 & 46 & 31.7 & & \\
\hline & 23 & & 241 & 46 & 19.6 & 0.7866 & 0.036 \\
\hline & 25 & & 340 & 46 & 7.8 & & \\
\hline & 27 & & $44^{\circ}$ & 45 & $5^{6.2}$ & 0.7905 & 0.035 \\
\hline & 29 & & 54 I & 45 & 44.9 & & \\
\hline \multirow[t]{2}{*}{ Juli } & I & & 642 & 45 & 33.9 & $0.794 \mathrm{I}$ & 0.034 \\
\hline & 3 & 8 & $7 \quad 44$ & +45 & $23 \cdot x$ & & \\
\hline
\end{tabular}

\begin{tabular}{|c|c|c|c|c|c|c|c|}
\hline \multicolumn{2}{|c|}{1905} & \multicolumn{2}{|l|}{$\alpha$} & \multicolumn{2}{|c|}{$\delta$} & $\log \Delta$ & \multirow[t]{2}{*}{$\mathrm{H}$} \\
\hline Juli & 3 & $8^{\mathrm{h}}{ }^{1} 7^{\mathrm{m}}$ & ${ }^{n} 44^{33}$ & $+45^{\circ}$ & $23^{\prime} .1$ & & \\
\hline & 5 & I 8 & 46 & 45 & т 2.6 & 0.7974 & 0.033 \\
\hline$\therefore$ & 7 & 19 & 48 & 45 & 2.3 & & \\
\hline & 9 & 20 & $5^{\circ}$ & 44 & $5^{2.3}$ & 0.8004 & $0.03^{2}$ \\
\hline & I I & 21 & 53 & 44 & 42.5 & & \\
\hline & I 3 & 22 & 56 & 44 & 33.0 & 0.8031 & $0.03 \mathrm{r}$ \\
\hline & I 5 & 23 & 59 & 44 & 23.8 & & \\
\hline & 17 & 25 & 2 & 44 & I 4.8 & 0.8056 & 0.031 \\
\hline & 19 & 26 & 5 & 44 & 6.0 & & \\
\hline & $2 I$ & 27 & 7 & 43 & 57.4 & 0.8078 & 0.030 \\
\hline & 23 & 28 & 9 & 43 & $49 . \mathrm{I}$ & & \\
\hline & 25 & 29 & I I & 43 & 41.1 & 0.8097 & 0.029 \\
\hline & 27 & 30 & I 3 & 43 & 33.3 & & \\
\hline & 29 & 31 & I 4 & 43 & 25.8 & 0.8114 & 0.029 \\
\hline & $3^{I}$ & $3^{2}$ & I 5 & 43 & I 8.5 & & \\
\hline Aug. & 2 & 33 & I 5 & 43 & 11.5 & $0.8 \times 28$ & 0.028 \\
\hline & 4 & 34 & ${ }^{1} 5$ & 43 & $4 \cdot 7$ & & \\
\hline & 6 & 35 & I 4 & 42 & 58.1 & 0.8 I 39 & 0.028 \\
\hline & 8 & $3^{6}$ & I 2 & $4^{2}$ & $5 \mathrm{x.8}$ & & \\
\hline & 10 & 37 & 9 & 42 & $45 \cdot 7$ & $0.8 \times 47$ & 0.028 \\
\hline & I 2 & 38 & 5 & $4^{2}$ & 39.9 & & \\
\hline & I 4 & 39 & 0 & 42 & $34 \cdot 3$ & $0.8 \mathrm{I} 53$ & 0.027 \\
\hline & I 6 & 39 & 54 & $4^{2}$ & 29.0 & & \\
\hline & I 8 & 40 & 48 & $4^{2}$ & 23.9 & 0.8157 & 0.027 \\
\hline & 20 & $4 \mathrm{I}$ & 40 & 42 & I $9 . \mathrm{r}$ & & \\
\hline & 22 & 42 & $3 I$ & 42 & I 4.6 & 0.8158 & 0.027 \\
\hline & 24 & 43 & $2 \pi$ & 42 & I0.3 & & \\
\hline & 26 & 44 & 9 & 42 & 6.3 & 0.8 I 56 & 0.026 \\
\hline & 28 & 44 & $5^{6}$ & $4^{2}$ & 2.5 & & \\
\hline & 30 & 45 & 42 & $4 r$ & 59.0 & $0.8 \times 5^{2}$ & 0.026 \\
\hline Sept. & I & 46 & 26 & $4 \mathrm{r}$ & $55 \cdot 7$ & & \\
\hline & 3 & 847 & 9 & $+4 I$ & $5^{2.7}$ & 0.8 I 45 & 0.026 \\
\hline
\end{tabular}

Utrecht, I905 April II.

Einheit der Helligkeit 1904 April ${ }_{7} 7\left(9^{\mathrm{m}}\right)$.

A. A. Nijland, F. v.d. Bilt.

\title{
Photographische Aufnahmen von kleinen Planeten.
}

I905 April $13 \quad 4^{\mathrm{h}} 38.7 \mathrm{~m}$. Z. Königstuhl. Beobachter $P$. Gïts.

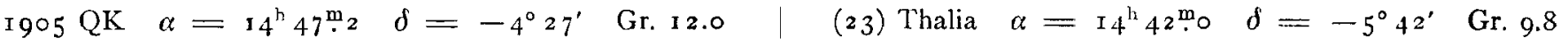

Astrophys. Institut Königstuhl-Hєidelberg, I 905 April I5.

M. IVolf.

(163) Erigone e (505) [1902 L]]: Effemeridi V.R. I. 26 accordano. (522) [1904 NC] non trovato (Effemeride A. N. 3995). E. Mitlosevich.

Komet 1905 a. März $287^{\mathrm{h}} 48^{\mathrm{ra}} \mathrm{I} 8^{\mathrm{s}}$ Washington m. t. $\alpha$ app. $=5^{\mathrm{h}} 5^{2^{\mathrm{m}} 0^{\mathrm{s}} 24} \delta$ app. $=+\mathrm{I} 3^{\circ} 39^{\prime} 30^{\prime \prime}$ I U. S. Nav. Observatory. Chester.

Inhalt zu Nr. 4orx. L. Terkán. Photometrische Beobachtungen veränderlicher Sterne in O-Gyalla. 33 . - P. Moschick. Bemerkungen zu dem Artikel »Helles Meteor von I904 März $21 \%$ in Astr. Nachr. 4008. 39. - Beobachtungen des Kometen I905 a. 39 . A. Alitti. Cometa 1905 a. 41. - F. Palisa. Beobachtungen von kleinen Planeten und Kometen. 43. - P. V. Neugebauer. Ephemeride des Planeten (498) Tokio. 43. - E. Stromgren. Ephemeride des Kometen I905 II (1904 e). 45 . - Elemente und Ephemeride des Kometen I905 a. 45. - A. A. Nijland, F. v. d. Bilt. Ephemeride des Kometen 1904 I. 47. - M. Wolf. Photographische Aufnahmen von kleinen Planeten. 47. - Kleine Mitteilungen. 47. 